\title{
Digitally designed holographic optical element for light field displays
}

\author{
Boaz Jessie Jackin, ${ }^{1, *}$ Lode Jorissen, ${ }^{2}$ Ryutaro OI, ${ }^{1}$ Jui YI Wu, ${ }^{3}$ Kokı Wakunami, ${ }^{1}$ Makoto Okui, ${ }^{1}$ \\ YasuyukI Ichihashl, ${ }^{1}$ Philippe Bekaert, ${ }^{2}$ YI Pal Huang, ${ }^{3}$ and Kenjl Yamamoto ${ }^{1}$ \\ ${ }^{1}$ National Institute of Information and Communications Technology (NICT), Tokyo 184-8795, Japan \\ ${ }^{2}$ Hasselt University-tUL, Wetenschapspark 2-3590 Diepenbeek, Belgium \\ ${ }^{3}$ National Chiao Tung University, 30010 Hsinchu, Taiwan \\ *Corresponding author: jackin@nict.go.jp
}

Received 11 April 2018; revised 4 July 2018; accepted 8 July 2018; posted 10 July 2018 (Doc. ID 328137 ); published 31 July 2018

\begin{abstract}
Concave micro-mirror arrays fabricated as holographic optical elements are used in projector-based light field displays due to their see-through characteristics. The optical axes of each micro-mirror in the array are usually made parallel to each other, which simplifies the fabrication, integral image rendering, and calibration process. However, this demands that the beam from the projector be collimated and made parallel to the optical axis of each elemental micro-mirror. This requires additional collimation optics, which puts serious limitations on the size of the display. In this Letter, we propose a solution to the above issue by introducing a new method to fabricate holographic concave micro-mirror array sheets and explain how they work in detail. 3D light field reconstructions of the size $20 \mathrm{~cm} \times 10 \mathrm{~cm}$ and $6 \mathrm{~cm}$ in depth are achieved using a conventional projector without any collimation optics. () 2018 Optical Society of America
\end{abstract}

OCIS codes: (120.2820) Heads-up displays; (050.1965) Diffractive lenses; (090.2890) Holographic optical elements.

https://doi.org/10.1364/OL.43.003738

Provided under the terms of the OSA Open Access Publishing Agreement

Electro-holography and light field displays are the probable candidates for the next-generation 3D display technology. Electro-holographic 3D displays require small pixels (submicron size) and processing of large pixel numbers, conditions which the currently existing "display devices" and "computing devices" fail to fulfill. On the other hand, the pixel specifications of light field displays fall within the capabilities of currently available display and computing devices. However, a light field display requires additional optical components, whereas holography does not require any optics for reconstruction. The most important optical component that determines the quality of a light field display is the micro-lens array sheet. The micro-lens array receives light rays from a $2 \mathrm{D}$ display and re-directs it in 3D space to reconstruct a scene with depth. The image being fed into the $2 \mathrm{D}$ display is a set of different perspective views of a particular scene (known as elemental images). The 2D display can be either an LCD (liquid crystal display) panel or a projector. In the LCD panel-based system, the lens array is sandwiched directly on top of the liquid crystal panel whereas, in a projector-based system, the projector projects the $2 \mathrm{D}$ elemental images onto the "microlens" or "concave micro-mirror" (hereafter simply represented as "micro-mirror") array kept at a distance. The advantages of a projector-based system compared to an LCD-based system are (1) its ability to display at larger size using a small display panel, (2) easy implementation of spatial/temporal multiplexing. Moreover, considering the see-through property, the LCD panelbased system always requires beam combining optics, while the projector-based system can realize it without the need for any combiner if HOE micro-mirror arrays are used $[1,2]$. The seethrough property is becoming more attractive due to the recent increase in augmented reality (AR) related contents. Hence, a projector and HOE micro-mirror array-based light field display system is the most likely candidate to serve AR requirements.

Various systems have been reported that are capable of delivering $3 \mathrm{D}$ content on a see-through screen $[3,4]$. These systems posses several advantages and disadvantages in comparison to each other, but there is one common issue that affects all of them. It is the necessity to use collimation optics between the projector and micro-mirror array to deliver a plane beam to the micro-mirror array. The usage of collimation optics gives rise to two major issues (with serious effects on the commercial success): (1) the volume, weight, and optical path length consumed by the collimation optics makes it very difficult to simplify the system and bring it to a form factor comparable to other existing techniques; and (2) the maximum display area of the projector and full size of the micro-mirror array cannot be utilized unless the aperture size of the collimation optics is also increased, which is very costly.

Figure 1(a) shows the top view, and Fig. 1(b) shows the side view of a typical projection-based light field display system. Figures 1(a) and 1(b) show the incident (blue lines) and diffracted (red lines) beams when no collimation optics is used. The collimation optics helps in collimating the diverging projection beam, thereby bringing the diffracted reconstruction beam back into the viewing zone [Fig. 1(c)]. The same applies to the vertical direction as well. In this Letter, we intend to replace the collimation optics through special modifications 

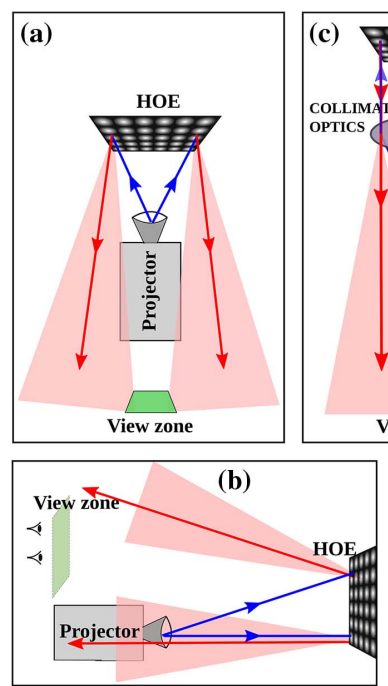

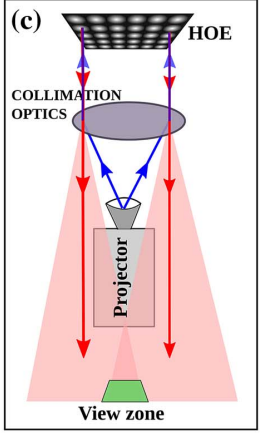

View zone

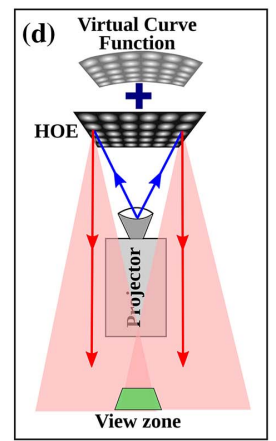

(e)

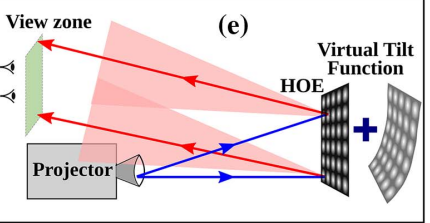

Fig. 1. Principal ray direction without collimation optics. (a) Top view and (b) side view. Principal ray direction with collimation optics. (c) Top view. Principal ray direction with virtual curve function. (d) Top view and (e) side view.

to the micro-mirror array, as shown in Figs. 1(d) and 1(e). The modification is nothing but a simple (variable) tilt function added to all the holographic micro-mirror elements in the array (both in the horizontal and vertical directions), so that the principle rays at the extreme edges are brought back into the viewing zone. To achieve this, novel changes have to be incorporated into the conventional micro-mirror array fabrication process.

The holographic micro-mirror array fabrication is conventionally done using a volume hologram recording setup. In the fabrication process shown in Fig. 2(a), an existing lens array is used as an object to record a hologram. This is a two-step process where a lens array is fabricated first (by other techniques such as casting, molding, etching, or laser beam writing) which is then used to generate the object beam in the second fabrication process (hologram recording). This can also be achieved in a single step by using a single microscopic objective lens to generate the object beam and then recording the micro-mirrors one by one with the help of an X-Y translation stage, as shown in Fig. 2(b). Such systems have been developed and reported recently [5]. It is important to notice that in all the HOE fabrication techniques explained earlier, a plane beam is used as reference, which demands the use of a plane beam (only) again for reconstruction. This is the root cause that necessitates collimation optics for which we propose a solution in this Letter. Before presenting our method, it is worth discussing other possible solutions.

The most straightforward solution will be to modify the fabrication/recording setup, shown in Fig. 2(a) by replacing the plane reference with a diverging reference beam (which resembles the divergence of the projector). However, this again becomes a two-step process that requires a lens array to be fabricated by other methods initially. Another solution will be to modify the recording setup shown in Fig. 2(b) and apply a variable tilt to the reference beam in both axes, but it makes the recording setup more complex and prone to errors. To the best of our knowledge, a successful implementation of the
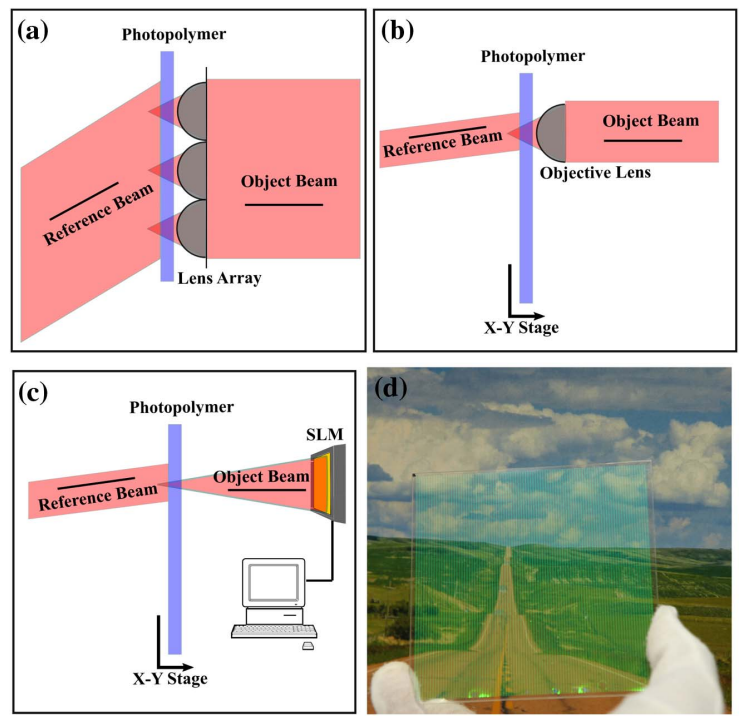

Fig. 2. Micro-mirror array fabrication methods (a) using an already existing lens array as an object and (b) using a microscopic objective lens as an object. (c) Proposed method and (d) a fabricated micromirror array on photopolymer.

above-mentioned ideas has not been reported yet due to these difficulties. Some of the difficulties can be overcome by considering modifications to the object beam or fabricated object (micro-mirror array) itself, instead of modifying the reference beam. Oshima et al. [6] have reported a technique where a flat micro-mirror array (object) is fabricated and then bent into a curved shape, which helped in increasing the view angle in the horizontal direction. However, this method has two drawbacks: (1) being a plane surface, the focal plane cannot deliver sharp 2D elemental images to the micro-mirror array (excluding a laser projector), and (2) calibration for alignment of projector pixels with a micro-mirror array in a curved plane is very complex. Instead of physically bending the micro-mirror array, the potential of holography can be utilized to realize the curvature virtually. Such an idea has been reported by Takahashi et al. [7] where a lens array and cylindrical lens have been used as the object in the recording process. The fabricated HOE has the property of both micro-mirror arrays and a horizontal curvature. However, here again the fabrication process requires an already fabricated lens array. The solution we propose is very simple and is shown in Fig. 2(c). We use a spatial light modulator to generate the object beam where we include all the micro-mirror + horizontal tilt + vertical tilt functions. The spatial light modulator (SLM) allows us the freedom to choose any combination of parameters (within the limits of SLM's space bandwidth) according to the reconstruction requirements. We call this fabricated micro-mirror array a digitally designed holographic optical element (DDHOE) as reported in one of our earlier works [8]. It takes $16 \mathrm{~h}$ to fabricate a micro-mirror array of the size $10 \times 10 \mathrm{~cm}$. A fabricated DDHOE micromirror array having $100 \times 200$ elemental micro-mirrors is shown in Fig. 2(d). The function of collimation optics, hence, is replaced with the included horizontal and vertical tilt functions, which significantly simplifies the display setup. The design and implementation of the method are explained below. 
It is first required to numerically compute the hologram data so that, when fed into an SLM, would generate the object beam consisting of concave mirror functions and the required horizontal and vertical tilt function. The amount of horizontal and vertical tilt strictly depends on the display system configuration. The light field display system that has been developed to test the proposed idea is shown in Figs. 3(a) and 3(b). It consists of a 2D projector (Sony-VPL-VW515) with a resolution of $4096(\mathrm{H}) \times 2160(\mathrm{~V})$ pixels, of which $4096(\mathrm{H}) \times 2048(\mathrm{~V})$ active pixels were used for the experiments. The lens of the projector was adjusted to achieve a short throw of $20 \mathrm{~cm} \times$ $10 \mathrm{~cm}$ at a distance of $50 \mathrm{~cm}$ from the projector. A Canon EOS 5D mark-II with $180 \mathrm{~mm}$ prime lens was used for calibration. The micro-mirror arrays were fabricated on the photopolymer material Bayfol HX-102 from Covestro. Figures 3(c) and 3(e) represent the direction of the principal rays with regard to the side and top views of the display system, respectively. The distance $(D)$ between the projector $(P)$ and DDHOE was set to be $533 \mathrm{~mm}$. A micro-mirror array of the size $193 \mathrm{~mm}\left(L_{x}\right) \times$ $96.8 \mathrm{~mm}\left(L_{y}\right)$, with $187(\mathrm{H}) \times 187(\mathrm{~V})$ concave mirrors, was used for the experiments. The elemental concave mirrors were rectangular and measured $1.03 \mathrm{~mm}(\mathrm{H}) \times 0.51 \mathrm{~mm}(\mathrm{~V})$ in size, each with a focal length of $2.15 \mathrm{~mm}$. The hologram pattern corresponding to the concave mirror function is computed analytically using focal length, wave length, and size information, which is nothing but a Fresnel zone plate. The horizontal curvature and vertical tilt functions are realized by applying corresponding $\mathrm{X}$ and $\mathrm{Y}$ shifts to the Fresnel zone plates. The viewing position is located behind the projector which is approximately $100 \mathrm{~cm}$ away from the DDHOE. The calibration camera was placed at the center of the viewing zone.
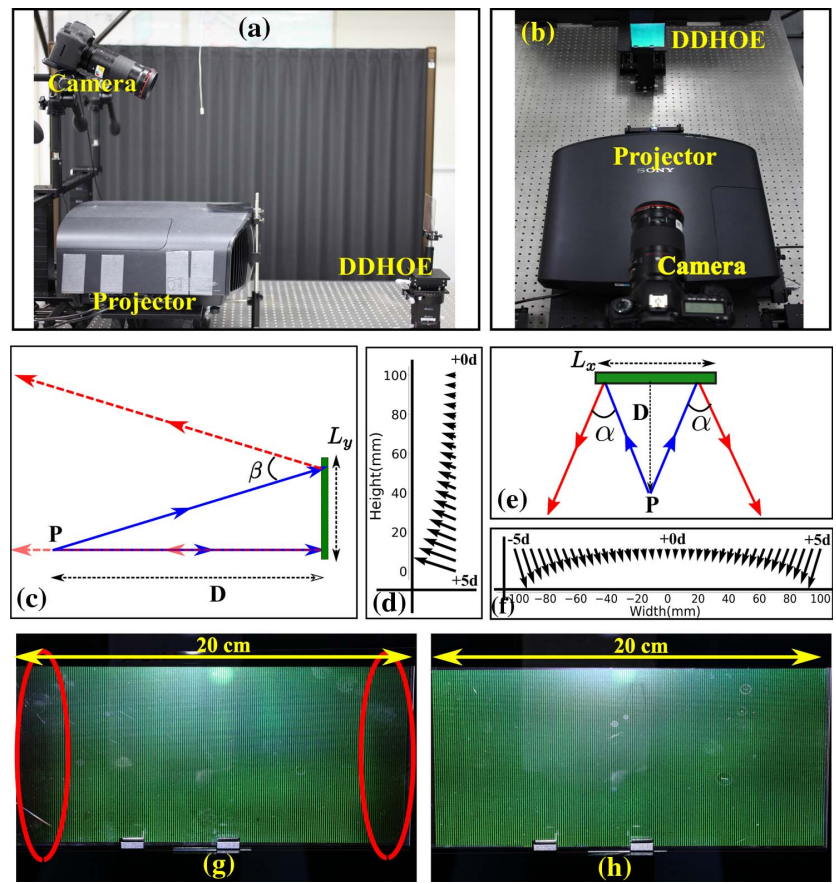

Fig. 3. Display system. (a) Side view and (b) top view. (c) Principal ray direction (side view). (d) Plot of the micro-mirror tilt in a vertical direction. (e) Principal ray direction (top view). (f) Plot of the micromirror tilt in a horizontal direction. (g) Fully lit DDHOE without a horizontal tilt, and (h) fully lit DDHOE with a horizontal tilt.
The maximum X-Y tilt required to bring all the rays back into the viewing zone is determined by the parameters $\alpha$ and $\beta$ shown in Figs. 3(c) and 3(e). The values of $\alpha$ and $\beta$ were calculated from the known (fixed) parameters $D, L_{x}$ and $L_{y}$ using simple trigonometry, and were found to be $20.60^{\circ}$. This means that with the help of horizontal and vertical tilt functions, the concave mirrors at the extreme edges should be able to bend the beam $10.3^{\circ}$ towards the center. Accordingly, a vertical tilt angle of $5.15^{\circ}$ was added to the bottom row of a micro-mirror array, which progressively decreases towards the top, as illustrated in Fig. 3(d). In Fig. 3(d), the y-axis represents the vertical dimension (height) of the DDHOE, the direction of the arrows corresponds to the direction of the tilt (not to scale), and the length of the arrows corresponds to the amount of the tilt from the normal to the DDHOE plane. Since this is a reflection screen, a tilt angle of $5.15^{\circ}$ will be able to produce a resultant tilt of $10.3^{\circ}$ at the viewer position. Similarly, in the horizontal direction, tilt angles of $-5.15^{\circ}$ and $+5.15^{\circ}$ are applied to the left- and rightmost column of the concave mirrors and then progressively decreased towards the center, where the column of the concave mirrors at the center suffer a tilt of $0^{\circ}$, as illustrated in Fig. 3(f). As can be seen, the amount of the tilt progressively changes, thereby forcing the diverging rays to diffract smoothly into the viewing zone, as shown earlier in Figs. 1(c) and 1(e).

The following experiment was conducted to evaluate the performance of tilt functions in compensating for collimation optics. Two micro-mirror array sheets were fabricated (with the above-mentioned specifications), one without the horizontal tilt function (LA-1) and the other with a horizontal tilt (LA-2). In order to understand the effects of the horizontal tilt function, the vertical tilt function was added to both micromirror arrays (LA-1 and LA-2). Figure 3(g) shows the image captured from the viewing zone when the LA-1 is completely illuminated, with all the pixels in the projector being ON. It could be observed that the left and right edges are dark (marked as red ellipses) which implies that the light diffracts away from the viewing zone, as explained earlier [Fig. 1(a)]. Figure 3(h) shows the corresponding image when LA-2 is illuminated. Uniform intensity distribution can be observed throughout, which indicates that light rays from all the micro-mirror elements were able to reach the viewing zone. The same applies to the vertical direction as well. Experimental results with and without a vertical tilt are not reported, because the DDHOE appears completely dark from the viewing zone without a vertical tilt. [Most of the beam goes back to the projector, as shown in Fig. 1(d).] The above experiment verifies that the function of collimation optics has been effectively replaced by adding appropriate functions to the DDHOE fabrication process.

Object data having two depth layers (I \& II) separated by a distance of $6 \mathrm{~cm}$, as shown in Fig. 4(a), were used for this purpose. The 3D scene consists of "hearts" and "flowers" as objects and are placed in such a way that the "flower" at the top-right (layer I) is located $3 \mathrm{~cm}$ in front of the DDHOE, and the one at the bottom-left is positioned $3 \mathrm{~cm}$ behind the screen (layer II) and vice-versa for the "heart" [Fig. 4(b)]. The depth of $3 \mathrm{~cm}$ was chosen based on practical observation of reconstruction quality, and a further increase in depth resulted in a visible blur. A calibration procedure was used to correct the rendered integral images to ensure proper alignment between projector pixels and DDHOE micro-mirrors [9]. Figures 4(d)-4(f) show reconstructions observed from the center, left, and right view 

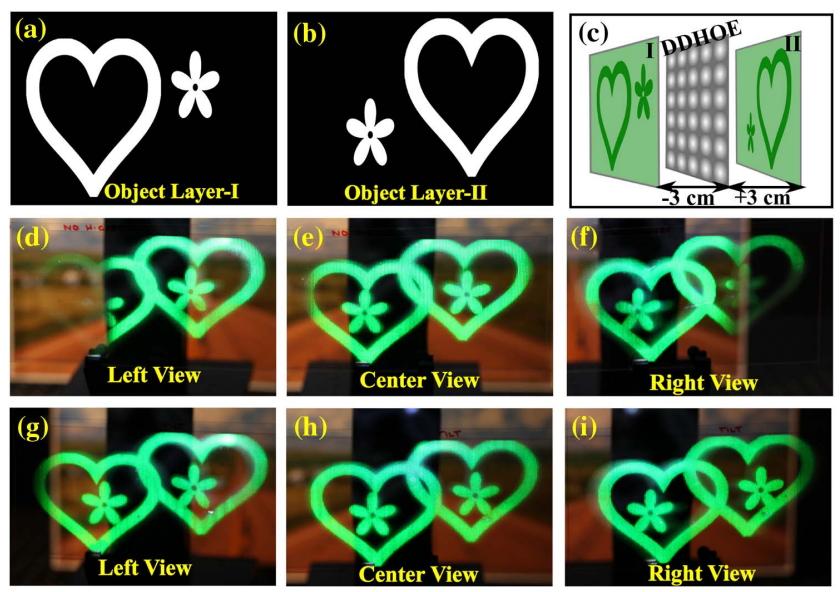

Fig. 4. (a) and (b) Object layers I \& II. (c) Position of the depth layers with DDHOE. Reconstructed views without a horizontal tilt. (d) Left, (e) center, and (f) right. Reconstructed views with a horizontal tilt. (g) Left, (h) center, and (i) right (Visualization 1).

positions, respectively, when the DDHOE without a horizontal tilt function (LA-1) was used. Figures 4(g)-4(i) correspond to observed reconstructions at the same positions, when the DDHOE with a horizontal tilt function (LA-2) was used. On comparison, it could be easily understood that $\mathrm{DDHOE}$ without a horizontal tilt loses reconstruction details at the edges when no collimation optics is used, whereas the proposed method alleviates the problem completely. Figure 4(i) also represents a video clip of the reconstruction captured by moving the camera along an arc in the viewing zone (Visualization 1). The maximum horizontal view angle was experimentally measured to be $10.37^{\circ}$. Even though the concave mirror tilts were not considered in the integral image rendering process, no errors were noticed in the reconstruction. However, it would be ideal to consider them in the ray tracing algorithm.

The following experiment demonstrates reconstruction capabilities with regard to 3D dynamic content. A 3D scene consisting of a volleyball court that is $10 \mathrm{~cm}$ in width and $6 \mathrm{~cm}$ in length was modeled in a computer [Figs. 5(a) and 5(b)]. The "net" in the scene is at the DDHOE plane, and the scene extends $3 \mathrm{~cm}$ in front and $3 \mathrm{~cm}$ behind the DDHOE. A DDHOE of $10 \mathrm{~cm} \times 10 \mathrm{~cm}$ with $187(\mathrm{~V}) \times 93(\mathrm{H})$ concave mirrors was used for this experiment. Figure 5(c) shows the reconstruction and represents a replay of the reconstructed scene captured by a camera moved along an arc in the viewing zone (Visualization 2). The maximum view angle for this $10 \mathrm{~cm}$ wide scene was measured to be $20.21^{\circ}$. The depth of the reconstructed scene can be verified by changing focus, as shown in Visualization 3 and represented by Figs. 5(d) and 5(e). Here the yellow smiley is a real object that is placed $3 \mathrm{~cm}$ in front of the DDHOE, and the red smiley is placed $3 \mathrm{~cm}$ behind the DDHOE. The defocused regions in the scene verify the scene depth of $6 \mathrm{~cm}$.

This Letter proposes a solution to the drawbacks associated with the necessity of collimation optics, in a large size seethrough light field display. The idea is to replicate the functions of collimation optics on the micro-mirror array itself. Fabrication of micro-mirror array as a DDHOE was used as the technique to realize the idea. Micro-mirror arrays of the

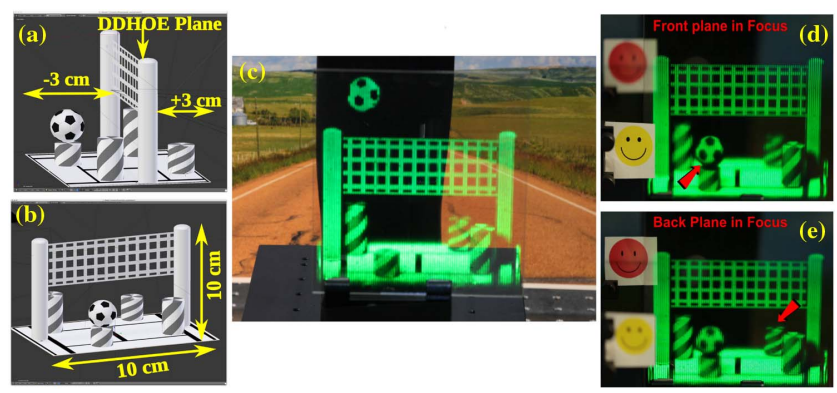

Fig. 5. Animated 3D scene. (a) Front view and (b) side view. (c) Reconstruction (Visualization 2). (d) Focused at a back plane and (e) focused at a front plane (Visualization 3).

size $20 \mathrm{~cm} \times 10 \mathrm{~cm}$ were fabricated as DDHOEs with carefully chosen design parameters according to system requirements. Reconstruction experiments using micro-mirror arrays with and without tilt functions verify the effectiveness of the proposed method. Reconstruction of a 3D animated scene demonstrates the dynamic capabilities of the display system. Using this method, a projector-based see-through light field system gets greatly simplified. The limitation of the proposed method is that the choice of focal length and maximum concave mirror tilt are limited by the space bandwidth of the SLM. However, currently available SLMs allow us to easily realize display systems up to $20 \mathrm{~cm}$ with a view angle of $10^{\circ}$. Using collimation optics to realize a display system of $20 \mathrm{~cm}$ will incur a larger space (path length), higher cost, and much greater weight. Organic light emitting diode displays are starting to be transparent and, hence, could substitute the projector sometime in the future. Hence, we believe this approach is very promising and will be investigated further to make see-through light field displays a commercial success. In the future, we intend to conduct a quantitative study to determine the maximum DOF and resolution of the reported system and to improve the fabrication technique to accommodate color reconstructions and larger view angles.

Funding. Japan Society for the Promotion of Science (JSPS) (16H01742, 18H03281, 26790064).

\section{REFERENCES}

1. K. Hong, J. Yeom, C. Jang, G. Li, J. Hong, and B. Lee, Opt. Express 22 , 14363 (2014).

2. K. Hong, J. Yeom, C. Jang, J. Hong, and B. Lee, Opt. Lett. 39, 127 (2013).

3. M. Yamaguchi and R. Higashida, Appl. Opt. 55, A178 (2016).

4. Q.-H. Wang, C.-C. Ji, L. Li, and H. Deng, Opt. Express 24, 9 (2016).

5. J. Jeong, C.-K. Lee, K. Hong, J. Yeom, and B. Lee, Appl. Opt. 53, G12 (2014).

6. Y. Oshima, H. Takahashi, and K. Yamada, Proc. SPIE 8648, 86481N (2013).

7. H. Takahashi, H. Fujinami, and K. Yamada, Proc. SPIE 6055, 60551C (2006).

8. K. Wakunami, P.-Y. Hsieh, R. Oi, T. Senoh, H. Sasaki, Y. Ichihashi, M. Okui, Y.-P. Huang, and K. Yamamoto, Nat. Commun. 7, 12954 (2016).

9. B. J. Jackin, L. Jorissen, R. Oi, K. Wakunami, Y. Ichihashi, M. Okui, P. Bekaert, and K. Yamamoto, Frontiers in Optics (Optical Society of America, 2017), paper JTu2A.105. 